\title{
An Oscillation-Based Model for the Neuronal Basis of Attention
}

\author{
ERNST NIEBUR, ${ }^{*} \dagger$ CHRISTOF KOCH,* CHRISTOPHER ROSIN*
}

Received 16 November 1992; in revised form 29 March 1993

\begin{abstract}
We propose a model for the neuronal implementation of selective visual attention based on the temporal structure of neuronal activity. In particular, we set out to explain the electrophysiological data from areas V4 and IT in monkey cortex of Moran and Desimone [(1985) Science, 229, 782-784] using the "temporal tagging" hypothesis of Crick and Koch [(1990a) Cold Spring Harbor Symposiums in Quantitative Biology, LV, 953-962; (1990b) Seminars in the neurosciences (pp. 1-36)]. Neurons in primary visual cortex respond to visual stimuli with a Poisson distributed spike train with an appropriate, stimulus-dependent mean firing rate. The firing rate of neurons whose receptive fields overlap with the "focus of attention" is modulated with a periodic function in the $40 \mathrm{~Hz}$ range, such that their mean firing rate is identical to the mean firing rate of neurons in "non-attended" areas. This modulation is detected by inhibitory interneurons in V4 and is used to suppress the response of V4 cells associated with non-attended visual stimuli. Using very simple single-cell models, we obtain quantitative agreement with Moran and Desimone's (1985) experiments.
\end{abstract}

Focal attention $40 \mathrm{~Hz}$ oscillations Temporal structure of neural signals Extrastriate cortex Monkey Computer modeling

\section{INTRODUCTION}

Selective attention is the process whereby a particular piece of information is selected from a sensory array for further processing, in particular for recognition or mnemonic tasks. Visual attention usually manifests itself within a single, circumscribed spatial area that can vary in size and that scans objects in the visual field at a rate of about $30-50 \mathrm{msec}$ per object. Visual attention has been postulated to operate by either suppressing all distracting information, by enhancing the selected (attended) information, or by both [for reviews of the psychophysical and cognitive literature on attention see Treisman (1988), Julesz (1991), Saarinen and Julesz (1991), Posner and Petersen (1990) and Kanwisher and Driver (1992)].

At the neuronal level, the cortical areas thought to be involved in the control and expression of attention include the object recognition pathway, in particular extrastriate areas V4 and IT, as well as the posterior parietal cortex and parts of prefrontal cortex. Among extra-cortical sites, the pulvinar nuclei of the thalamus and the superior colliculus in the midbrain have been implicated in the control of attention [for reviews of the neuroanatomy and neurophysiology of attention see Colby (1991), Posner and Petersen (1990) and Posner and Driver (1992); for models of attention see Koch and Ullman (1985) and Olshausen, Anderson and van Essen (1993)].

\footnotetext{
*Computation and Neural Systems Program, California Institute of Technology, Pasadena, CA 91125 , U.S.A. $\dagger$ To whom all correspondence should be addressed.
}

How attention acts at the level of single neurons in extrastriate cortex has been elucidated by Moran and Desimone (1985) and Desimone, Wessinger, Thomas and Schneider (1991). When two different objects, say a red and a green bar, are both located within the receptive field of a V4 neuron selective for red, the neuron will respond vigorously if the monkey attends to the red stimulus, but respond much less if the monkey is attending to the green stimulus. The stimulus is identical in both cases (a red and a green bar); the difference is only in the internal state of the monkey. The Moran and Desimone (1985) effect is mainly suppressive, since the response of the cell to the attended stimulus does not increase significantly over its response if the monkey is attending to a stimulus outside the receptive field. In other words, the receptive field of such a V4 cell shrinks around the attended stimulus.

Recently, Crick and Koch (1990a, b) outlined a neurobiological theory of awareness. One of the key points of this theory is the hypothesis that selective visual attention serves to rapidly and transiently bind the neural activity of cells in the many different visual areas throughout the cortical system, providing the system (i.e. the observing subject) with a unitary percept of the currently viewed object in the visual field. Crick and Koch (1990b) proposed that selective visual attention manifests itself at the single cell level via "temporal tagging", that is that attention modulates the detailed temporal structure of the neuronal discharge at the level of primary visual cortex in response to a visual stimulus. This modulation affects only the temporal structure of 
the spike trains of a $\mathrm{Vl}$ neuron responding to an attended or non-attended stimulus, not its mean firing rate, in agreement with neurophysiology. It is possible that "temporal tagging" is mediated by the synchronized oscillatory neuronal activity in the $25-60 \mathrm{~Hz}$ range (referred to as " $40 \mathrm{~Hz}$ " oscillations) observed in the visual system of the cat and macaque monkey (Gray \& Singer, 1989; Eckhorn, Bauer, Jordan, Brosch, Kruse, Munk \& Reitboeck, 1988; Kreiter \& Singer, 1992; Livingstone, 1991; see however, Tovee \& Rolls, 1992; Young, Tanaka \& Yamane, 1992 for studies failing to find evidence for such oscillations in areas V1, MT and IT of the behaving monkey).

In the present work, we tested these ideas by studying a model of an attentional system which uses oscillatory firing activity for the neural implementation of focal attention. We presume the existence of a "saliency map" à la Koch and Ullman (1985) (see also Treisman, 1988) which encodes information on where salient (conspicuous) objects are located in the visual field, but not what these objects are. Saliency is here meant to be understood in terms of simple operations, implemented by center-surround type of operations, i.e. a green object among many red ones or a moving stimulus in an otherwise stationary scene would constitute very salient objects. Because in general only a single object can be attended at any point in time, the topographic saliency map has to sclcct, by some kind of winnertake-all mechanism, the currently most conspicuous region in the visual field and direct attention to it. After a short time, this location becomes inhibited in the saliency map and attention switches to the next most conspicuous location.

Possible anatomical sites for this topographic map include the superior colliculus and the dorsomedial region of the pulvinar (Robinson \& Petersen, 1992); there is also evidence for multiple or distributed maps (Desimone, Wessinger, Thomas \& Schneider, 1989). In the present work, we assume that salient objects have been selected in the visual field by such a mechanism. How can such a signal now be used to lead to the changes in receptive fields observed by Desimone and his colleagues. According to an idea expressed by Desimone (1992), competition between cells in area V4 (e.g. by mutual inhibition) could be biased in favor of cells representing attended stimuli. Our basic hypothesis is that this is accomplished using different temporal structures (but identical average spike rates) of the spike trains generated by neurons inside and outside the focus of attention, thereby "tagging" or "labcling" all neurons in primary visual cortex whose receptive field (partially) overlaps with the spotlight of attention. Signals along the tagged pathway compete-at the level of V4 and beyond-with signals in the untagged pathway, leading to an inhibition or reduction in the response of neurons in the untagged pathway.

\footnotetext{
*We are here not concerned with the issue of how this color or hue-selectivity is obtained, but only in the action of attention. Our results do not depend on the simplification of using only two features.
}

We here present a model where the temporal tagging (Crick \& Koch, 1990b) is implemented at the single cell level using an oscillatory signal in the $30-50 \mathrm{~Hz}$ range. We have also investigated to what extent temporal tagging can be achieved by synchronizing the firing rate of a group of neurons without any need to invoke oscillations (Niebur \& Koch, 1993). These models lead to experimentally testable predictions. We have also to assure that our model is consistent with known experimental data. In particular, it has been found that attentional modulation does not change the level of activity in V1 cells. In area V4, Moran and Desimone's data show that (1) a cell's response to a single stimulus in its receptive field is the same whether the stimulus is attended or not, (2) when a preferred and a non-preferred stimulus are present simultaneously in the receptive field of a cell, the cell's response will be suppressed if attention is directed at the non-preferred stimulus but unchanged if attention is directed at the preferred stimulus, (3) no such suppression of activity is observed when the attended stimulus is outside the receptive field of a cell, even if the distance to the unattended stimulus is the same as in case (2). Furthermore, our model should not be in contradiction to the vast body of experimental "common-sense". For instance, an oscillation-based model which would require levels of modulation that high that they could not have failed to be noticed in the course of standard electrophysiological experiments would have to be discarded.

\section{THE MODEL}

The gross architecture of our model is shown in Fig. 1(A). Input from the two-dimensional retina is fed via the lateral geniculate nucleus (LGN; not shown) into area V1, where the attentional modulation-originating in the saliency map in subcortical areas-is addcd. We assume that cells in VI are only selective to one of two different features which we consider to be the colors red or green.* The output of our model VI directly projects into our model V4 (neglecting possible pathways involving V2). Here it excites two sets of neurons, excitatory pyramidal cells as well as frequency-sensitive inhibitory interneurons. These interneurons inhibit pyramidal cells with opposite feature selectivity [Fig. 1(B)]. Since temporal modulation is restricted to cells in V1 within the spotlight of attention, the interneurons will detect this frequency. modulation and will inhibit the response of directly overlapping pyramidal cells to the non-attended stimulus, mimicking the Moran and Desimone (1985) experiments.

In the following we describe each of these elements in more detail. For the sake of simplicity of language, we will sometimes refer to neurons whose receptive fields are inside the focus of attention as "attended neurons" and to neurons which are sensitive to red or green stimuli as "red" or "green" neurons, respectively. 
(A)

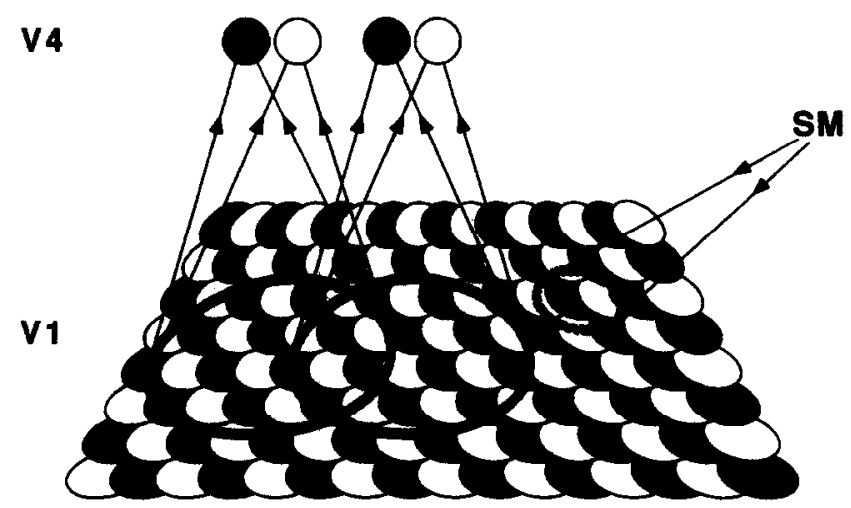

(B)

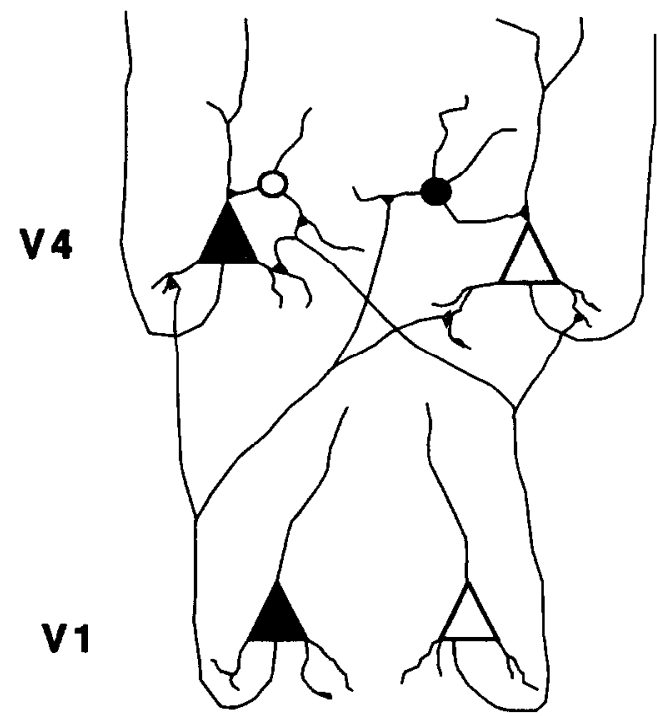

FIGURE 1. (A) Architecture of the model. Receptive fields of $\mathrm{Vl}$ cells are represented by overlapping circles arranged in a two-dimensional array (actual receptive fields in the model are square). White and gray circles represent cells receptive to the two features considered (referred to as red and green in the text). Actual overlap is larger than shown in the figure: every point is represented in the receptive fields of four cells of each feature type (i.e. by four red and four green cells). Open black circles denote the receptive fields of V4 cells, i.e. all green cells in a stack in V4 receive input from all V1 cell in the corresponding circle (arrows) and analogously for red cells [see (B) for details of the connectivity]. The shaded open circle indicates the focus of attention; the activity of all V1 cells inside this circle is subjected to oscillatory modulation by the saliency map (SM). (B) Schematic connectivity of the model. The two types (white and gray, representing red and green) of V1 pyramidal cells (triangles) project to a stack of cells in V4. Strong excitatory synapses are made to pyramidal cells (triangles) and smooth interneurons (circles) with the same preferred color, weaker connections are made to pyramidal cells of the other color. The smooth interneurons project with inhibitory synapses on pyramidal cells of the other color.

\subsection{Striate cortex}

The visual input into each Vl cell is provided by a $10 \times 10$ array of pixels. Cells have overlapping receptive fields with a coverage factor of 4 , i.e. every point in the visual field is represented within four receptive fields. The output of any V1 cell are action potentials, generated using a Poisson process of mean firing rate $\lambda$ in combination with a refractory period $\tau_{r}$, which is chosen randomly from a uniform distribution with values between 2 and $5 \mathrm{msec}$. That is, within any given interval of the small length $\Delta t$, the neuron will fire an action potential with probability $p_{1}=\lambda \Delta t$ if its last spike was more than $\tau_{\mathrm{r}}$ in the past and will otherwise remain silent.* Apart from this we assume no other temporal dependencies. Bair, Koch, Newsome and Britten (1993), analyzing 216 cells recorded from extrastriate area MT in the awake and behaving monkey, showed that the stochastic properties of about one-third of these cellsfiring at high discharge frequencies - can best be described on the basis of a Poisson process in combination with a short refractory period (see also Softky \& Koch, 1993).

The total firing rate $\lambda$ of any neuron is the sum of the spontaneous firing rate $\lambda_{\text {spont }}$, with $\lambda_{\text {spont }}=2$ spikes per sec, and the stimulus-dependent rate $\lambda_{0}$. This rate is determined by the properties of the stimulus. If no

*This is achieved by generating a random number $r$, uniformly distributed between 0 and 1 ; if $r \leqslant p_{1}$ and if the cell did not spike within the previous $\tau_{\mathrm{r}} \mathrm{msec}$, an output spike is generated. preferred stimulus is present (i.e. either a non-preferred stimulus or no stimulus at all) in the receptive field of the cell under study, $\lambda_{0}=0$. If a preferred stimulus is present, $\lambda_{0}$ is chosen to be proportional to the degree of spatial overlap of the receptive field of the cell with the stimulus,

$$
\lambda_{0}=\lambda_{\max } \times \text { overlap (stimulus, receptive field) }
$$

where $\lambda_{\max }=200$ spikes $\mathrm{sec}^{-1}$ and the overlap varies between zero (no overlap) and unity (complete overlap). Thus, the maximal firing rate of any cell is $\lambda_{\max }+\lambda_{\text {spont }}$ ( $202 \mathrm{~Hz}$ in this study).

The action of attention is to modulate this discharge without affecting its mean rate $\lambda$. We achieve this by using an inhomogeneous Poisson process, whose mean rate $\lambda(t)$ varies over time and is given by

$$
\lambda(t)=\lambda_{0}(1+A \sin (\omega t+\phi))+\lambda_{\text {spont }}
$$

where $\phi$ is an arbitrary, time-independent phase shift. The modulating frequency $\omega / 2 \pi$ which is imposed by the saliency map (see below) is assumed to be in the $25-60 \mathrm{~Hz}$ range ( $\gamma$ range). The parameter $A$, determining the strength of the modulation, is proportional to the spatial overlap of the receptive field of the cell with the focus of attention signal from the saliency map:

$$
A=A_{\max } \times \text { overlap }
$$

(focus of attention, receptive field)

where $A_{\max }=0.75$ and therefore $A \epsilon[0,0.75]$. The mean firing rate $\bar{\lambda}$, averaged over times longer than $2 \pi / \omega$, is 
always $\lambda_{0}+\lambda_{\text {spont }}$, independent of the level of attentional modulation.

We would like to emphasize at this point that, although $A=1$ is the largest value possible in equation (2) [due to the fact that $\lambda(t) \geqslant 0$ at all times], there would be a substantial stochastic component in the firing rate even for this value. This can be seen, for instance, in the power spectrum [not shown, but similar to the spectrum for $A_{\max }=0.75$ shown in Fig. 8(B)], where only a fraction of the total power is concentrated in the vicinity of $\omega$.

\subsection{The saliency map}

The task of the saliency map is to select the most salient parts of the visual field at any one time. Here we are not concerned with the selection mechanism (see Koch \& Ullman, 1985 for that) but with the mechanisms by which the result of the selection process is communicated to the information-processing occipito-temporal pathway. It will be our assumption that subcortical structures impose an oscillatory structure on the neural signals generated in the various visual cortices. In order to simplify matters somewhat, we here assume that this modulation only occurs at the level of primary visual cortex V1. The main candidate for such subcortical structures are the efferent connections from the different visual maps present in the pulvinar nuclei of the thalamus (inferior, lateral and medial) into striate and extrastriate cortex [for the neuroanatomy of the pulvinar and its relationship to saliency see Garey, Dreher and Robinson (1991) and Robinson and Petersen (1992)]. On the basis of inactivation and PET studies, parts of the pulvinar are known to be involved in the control of attention (LaBerge \& Buchsbaum, 1990; Petersen et al., 1987; Desimone et al., 1989; Kubota, Morimoto, Kanaseki \& Inomata, 1988).

We are here not concerned with a detailed, biophysically plausible, mechanism by which this periodic modulation is forced upon the V1 neurons; this could be implemented, for instance, by oscillatory subthreshold input to the Vl neurons. The inferior pulvinar projects onto processes in the superficial layers of striate cortex (Jones, 1985). These dendrites are among the most distal dendrites for most pyramidal cells, which is in agreement with their possible role as receiving modulatory input. It has been observed in slices that synaptic input at these distal sites can have considerable influence on the response of pyramidal cells, to the point of being sufficient to fire cells in the deep layer V (Cauller \& Connors, 1992). These authors also found that the synaptic input is mediated by fast, non-NMDA synapses (although some cells show an additional, long-lasting NMDA mediated depolarization), compatible with their possible function of conveying temporal modulation signals.

\subsection{Extrastriate cortex}

Following Crick and Koch (1990b), we assume that selective attention activates competition within a "stack" or microcolumn of neurons in V4, selective to a variety of different stimuli (each stack consists of a set of neurons representing all stimulus features and sharing similar receptive fields). Thus, in the presence of multiple stimuli, the neurons responding to the different stimuli will compete against each other. Because we assume that attention "labels" neurons in VI within a particular spatial location, the postsynaptic targets of these "labeled" neurons will win out in the competition at the level of V4.

In particular, we here presume the existence of frequency-selective inhibitory interneurons (stellate cells) in V4. These interneurons are assumed to act like bandpass filters, selective to spikes arriving every $25 \mathrm{msec}$ or so. Thus, $40 \mathrm{~Hz}$ synaptic input optimally excites these cells, while higher or lower input frequencies cause a smaller response. These interneurons inhibit the pyramidal cells of opposing response selectivity [Fig. 1(B)]. For instance, if the output of $\mathrm{V} 1$ cells responding to red are frequency modulated, their postsynaptic interneurons in V4 will inhibit the response of the green pyramidal cell in V4. Because green cells in V1 are not frequency-modulated (they are outside the spotlight of attention), the associated V4 inhibitory interneurons will respond only little. Thus, in the competition among red and green pyramidal cells in V4, red will win out.

We here assume that each V4 neuron, whether pyramidal or stellate cell, receives overlapping input from 100 $\mathrm{V} 1$ cells. Thus, the receptive fields of V4 cells is much larger than those of $\mathrm{V} 1$ cell. We assumed an axonal delay of $5 \mathrm{msec}$ between cells in different areas and neglected any delay within each area.

2.3.1. Inhibitory interneurons. Llinas, Grace and Yarom (1991) recorded in the cortical slice preparation neurons optimally selective to intracellular current steps in the $10-50 \mathrm{~Hz}$ range. Because the neurons are located in layer IV, and are small, with smooth, aspiny dendrites, Llinas et al. (1991) argue that they correspond to inhibitory interneurons. Functionally, such cells can be thought of as bandpass filters, similar to the frequencyselective haircells of the bullfrog, turtle or lizard cochlea (Crawford \& Fettiplace, 1981; Fettiplace, 1987).

The electrical behavior of these cells is usually modeled by describing the different ionic currents underlying their behavior using a Hodgkin-Huxley like formalism (Yamada, Koch \& Adams, 1989). Although this approach is quite general and powerful it is computationally expensive and requires detailed knowledge of the relevant channel kinetics. A time-honored simpler method consists in linearizing the system of equations around the resting potential. Conceptually, this can be thought of as replacing the voltage-dependent ionic conductances associated with the different ion channels by resistances, capacitances and inductances [Fig. 2(A)]. One now obtains, both in the case of the original Hodgkin-Huxley equations (Mauro, Conti, Dodge \& Schor, 1970) as well as for the cochlear haircells a linear system with a bandpass behavior [Fig. 2(B)], with the resonance frequency varying from a few to several hundred Hertz, depending on the density and kinetics of the various currents (Crawford \& Fettiplace, 1981; Hudspeth \& Lewis, 1988a). Such bandpass behavior, albeit 
(A)

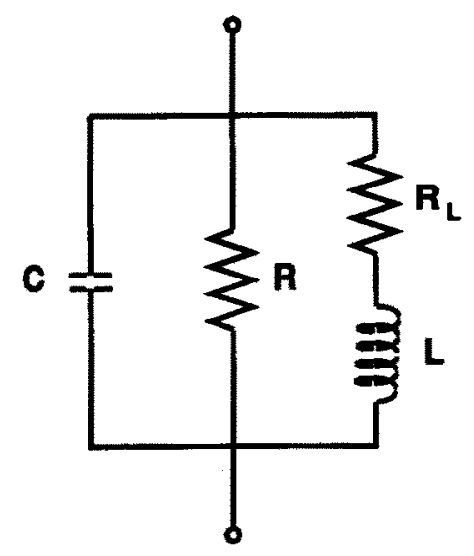

(B)

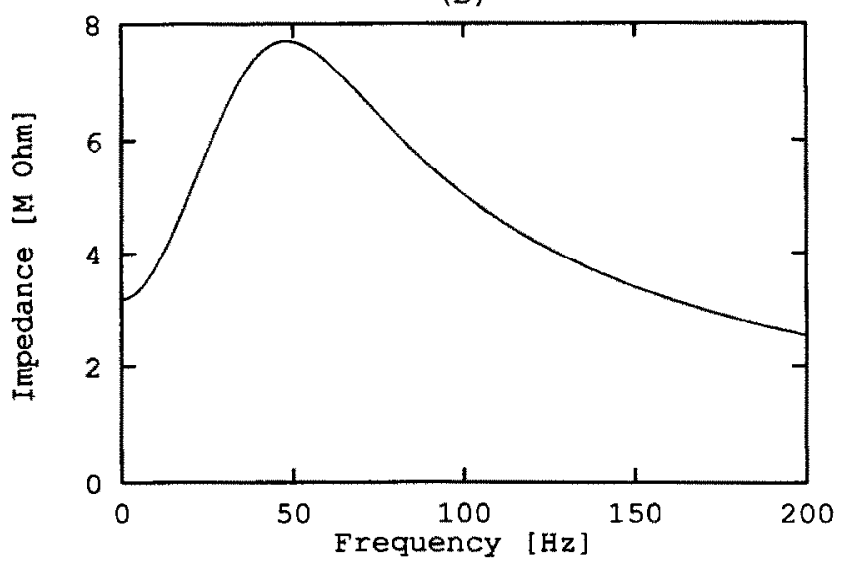

FIGURE 2. (A) Equivalent electrical circuit of a neuron with quasiactive membrane. The behavior of this circuit is characterized by equation (5). (B) Impedance (in $\Omega$ ) of the circuit shown in (A) as a function of the frequency (in $\mathrm{Hz}$ ) of the applied current. The resonance frequency is close to $40 \mathrm{~Hz}$. The behavior shown is very similar to that of linearized Hodgkin-Huxley membrane (Koch, 1984).

at lower frequency, has also been observed in neocortical neurons (Hutcheon \& Puil, 1992). Such a linearized membrane description containing inductances (giving rise to a non-passive behavior) is sometimes termed quasiactive (Koch, 1984). Because knowledge of the ionic currents in the frequency-selective interneurons of Llinas et al. (1991) is lacking and we are in any case interested in the generic behavior of such circuits (rather than in the detailed biophysics of the voltage response) we choose to model our V4 interneurons using such a well-established linearized description (Fig. 2).

We assume that each action potential from the V1 neurons into these interneurons gives rise to an exponentially decaying current input, described by the differential equation

$$
\frac{\mathrm{d} I}{\mathrm{~d} t}=-\frac{I}{\tau_{I}}+\alpha \sum_{j} \delta\left(t-t_{j}\right)
$$

\footnotetext{
*Note that this is a conservative assumption; the observed frequency tuning of cells in the auditory system is much sharper (Fettiplace, 1987). Our intention here is to show that even relatively unspecific mechanisms are sufficient to make our model work.
}

where $\tau_{I}=5 \mathrm{msec}$ corresponds to the decay time of the synaptic event and the sum includes all synaptic inputs into the cell occurring at time $t_{j}$. The constant $\alpha=10^{-6}$ determines the size of the excitatory synaptic potential (EPSP). For our values of $\alpha$ and of the resting conductivity (see below), an individual synaptic event leads to a voltage change of a fraction of a millivolt, in agreement with experimental observations.

Following Koch (1984), we model the quasi-active membrane of the interneurons using a three branch RLC circuit [Fig. 2(A)]. The associated equation, describing the change in potential $V_{i}(t)$ in response to synaptic current $I(t)$ is

$$
\begin{aligned}
\frac{\mathrm{d}^{2} V_{\mathrm{i}}(t)}{\mathrm{d} t^{2}} & +\left(\frac{1}{R C}+\frac{R_{L}}{L}\right) \frac{\mathrm{d} V_{\mathrm{i}}(t)}{\mathrm{d} t} \\
& +\left(\frac{1}{L C}+\frac{R_{L}}{R L C}\right) V_{\mathrm{j}}(t) \\
= & \frac{1}{C} \frac{\mathrm{d} I(t)}{\mathrm{d} t}+\frac{R_{L}}{L C} I(t) .
\end{aligned}
$$

We here use $R=3 \mathrm{k} \Omega, C=1 \mu \mathrm{F}, L=15 \mathrm{H}$ and $R_{L}=1.5 \mathrm{k} \Omega$, to obtain a resonance frequency close to $40 \mathrm{~Hz}$ with a $\mathrm{Q}$ factor (the quotient of the impedances at the resonance frequency and at zero frequency) of about 2.5 [Fig. 2(B)].* For the standard Hodgkin-Huxley squid axon membrane, one obtains a resonance frequency of about $67 \mathrm{~Hz}$ and a $Q$ factor of about 2.8 (Mauro et al., 1970; Koch, 1984).

An all-or-none output pulse (spike) is generated if the intracellular voltage $V$ exceeds the time-dependent threshold $\Theta(t)=\Theta_{0}+1.5 \Theta_{i}(t)$ with $\Theta_{0}=10 \mathrm{mV}$. The time-dependent part of the threshold, $\Theta_{i}(t)$, is determined as a gliding average with a time constant $\tau_{\theta}=20 \mathrm{msec}$,

$$
\frac{\mathrm{d} \Theta_{\mathrm{i}}(t)}{\mathrm{d} t}=\frac{V_{\mathrm{i}}(t)-\Theta_{\mathrm{i}}(t)}{\tau_{\theta}}
$$

After emitting a spike, the cell's voltage is reset by subtracting $\Theta$ from $V_{\mathrm{i}}$ (this corresponds to resetting the voltage to zero in neurons with fixed thresholds). Cells have an absolute refractory period $\tau_{\mathrm{r}}$ chosen again randomly from the interval $[2 \mathrm{msec}, 5 \mathrm{msec}]$, i.e. a cell will not fire if its last spike occurred less than $\tau_{\mathrm{r}}$ ago.

2.3.2. Pyramidal cells. The interneurons in our simulated area V4 are presynaptic to excitatory V4 neurons which also receive direct input from V1 neurons and which are implemented as leaky integrate-and-fire neurons. Synaptic input is modeled in form of conductance changes with different time-courses and reversal potentials for excitatory and inhibitory synapses.

The potential $V_{\mathrm{e}}$ of an excitatory neuron is determined by

$$
\begin{aligned}
\tau_{\mathrm{m}} \frac{\mathrm{d} V_{\mathrm{e}}(t)}{\mathrm{d} t}=-V_{\mathrm{e}}(t)+ & \rho_{\mathrm{e}}(t)\left(E_{\mathrm{e}}-V_{\mathrm{e}}(t)\right) \\
& +\rho_{\mathrm{i}}(t)\left(E_{\mathrm{i}}-V_{\mathrm{e}}(t)\right)+\eta(t)
\end{aligned}
$$


where the first term of the right-hand side represents the transmembrane leakage current, the next two terms the time-dependent synaptic inputs and $\eta(t)$ is added noise. In the following, we will describe each of these terms in detail.

The leakage current is determined by the membrane time constant $\tau_{\mathrm{m}}=5 \mathrm{msec}$. This small value takes into account the influence of neuronal background activity on the membrane parameters for the spontaneous activity we assume (about 4 spikes per sec; see Table 1; Bernander et al., 1991).

All synaptic terms are products of a synaptic conductivity $\rho$ and the difference between $V_{\mathrm{e}}(t)$ and the respective reversal potential. In the case of excitatory input, the reversal potential was set to $E_{\mathrm{e}}=100 \mathrm{mV}$, in the case of inhibitory input, to $E_{\mathrm{i}}=-25 \mathrm{mV}$ (both relative to the resting potential, which we here normalize to 0 ). Excitatory synapses are assumed to be glutaminergic, of the quisqualate or kainate (non-NMDA) subtypes and therefore rapidly acting. We model their time course by a decaying exponential with a synaptic time constant $\tau_{\text {exc }}=1.5 \mathrm{msec}$,

$$
\begin{aligned}
\frac{\mathrm{d} \rho_{\mathrm{e}}(t)}{\mathrm{d} t}=-\frac{\rho_{\mathrm{e}}(t)}{\tau_{\mathrm{exc}}}+W_{\mathrm{e}} \sum_{j} \delta\left(t-t_{j}\right) & \\
& +\frac{W_{\mathrm{e}}}{4} \sum_{\mathrm{k}} \delta\left(t-t_{k}\right)
\end{aligned}
$$

where $t_{j}$ are the spike times of $\mathrm{V} 1$ with the same preferred color as the pyramidal neuron under study and $W_{\mathrm{e}}$ is a synaptic weight chosen as $W_{\mathrm{e}}=0.025$. The first sum runs over all spike times $t_{j}$ of the excitatory presynaptic neurons in Vl which are sensitive to the same color (red or green) as the pyramidal neuron under study. The second sum runs over the spike arrival times $t_{k}$ of the excitatory presynaptic neurons in $\mathrm{V} 1$ which are sensitive to the opposing color. The physiological reason for this contribution is the fact that the tuning curves of most neurons are not completely sharp. For instance, a redsensitive neuron may respond to some extent to visual input with a color other than red. Obviously, this response should be weaker than the response to a preferred stimulus, which is why the weight of thesc synapses is only $W_{\mathrm{e}} / 4$. For this choice, appearance of a nonpreferred stimulus in the receptive field of a cell enhances its firing rate by about 5-10\% (see Table 1).

We assume that inhibition is mediated by GABAergic

TABLE 1. Mean firing rates of excitatory V4 cells for different stimulus conditions (averaged over $10 \mathrm{sec}$ of simulated time)

\begin{tabular}{lcc}
\hline Stimulus type & Red cell firing rate & Green cell firing rate \\
\hline No attention & & \\
None (spont. act.) & 4 & 4 \\
Red & 47 & 7 \\
Green & 7 & 47 \\
Red and green & 50 & 50 \\
Attention on red stimulus & & \\
Red & 45 & 1 \\
Green & 6 & 47 \\
Red and green & 50 & 12 \\
\hline
\end{tabular}

synapses. GABA activates as well $\mathrm{GABA}_{\mathrm{A}}$ as well as $\mathrm{GABA}_{\mathrm{B}}$ receptors simultaneously, giving rise to an inhibition lasting between 10 and $100 \mathrm{msec}$. The time-course of inhibition is modeled as a decaying exponential with $\tau_{\text {inh }}=40 \mathrm{msec}$, such that

$$
\frac{\mathrm{d} \rho_{\mathrm{i}}(t)}{\mathrm{d} t}=-\frac{\rho_{\mathrm{i}}(t)}{\tau_{\mathrm{inh}}}+W_{\mathrm{i}} \sum_{l} \delta\left(t-t_{l}\right)
$$

with a weight $W_{\mathrm{i}}=40$. The sum runs over the spike times $t_{l}$ of the inhibitory V4 interneurons with oppposing color preference. The inhibitory weight $W_{i}$ has to be considerably larger than the excitatory weight $W_{\mathrm{e}}$ in our model, given the large number of afferent excitatory $\mathrm{Vl}$ connections. Furthermore, inhibitory synapses on pyramidal cells occur preferably at or close to the soma and are expected to have a stronger effect than more distally placed excitatory synapses.

The term $\eta(t)$ in equation (7), representing the spontaneous activity of the neuron caused by internal noise and background synaptic activity, is implemented as Gaussian noise with mean zero and standard deviation $2 \times 10^{-4}$. This value was chosen to obtain a spontaneous activity of about $4-5 \mathrm{~Hz}$, in agreement with experimentally observed values for V4 neurons (R. Desimone, personal communication).

The instant at which $V_{\mathrm{e}}(t)$ exceeds the threshold $\Theta_{\mathrm{e}}=10 \mathrm{mV}$, an output spike is generated and $V_{\mathrm{e}}$ is reset to zero (assuming that the last spike was generated prior to the refractory period $\tau_{r}$, chosen randomly from the interval [ $2 \mathrm{msec}, 5 \mathrm{msec}]$ ).

\section{RESULTS}

The activity of our V1 and V4 neurons is shown in Figs 3 and 4. Figure 3(A) shows the spontaneous and the visually evoked activity in a $\mathrm{V} 1$ cell whose receptive field is outside the focus of attention $[A=0$ in equation (2)], while Fig. 3(B) illustrates the change in activity when it receives an attentional modulation from the saliency map $\left[A=A_{\max }=0.75\right.$ in equation (2)]. A key aspect of Crick and Koch's (1990b) "temporally tagging" hypothesis is evident here: the action of attention does not lead to a change in the mean firing rate (the rate is $24.9 \mathrm{~Hz}$ in Fig. 3(A) and $23.5 \mathrm{~Hz}$ in Fig. 3(B), in each case for an average over $0.1 \mathrm{sec}$ of visual stimulation and $0.9 \mathrm{sec}$ of spontaneous activity), but to a frequency modulation not readily apparent in the spike trains.

To illustrate the effect of the competition between inhibitory populations, Fig. 4 shows the spike activity of two excitatory V4 neurons which receive input from unattended stimuli and attended stimuli, respectively. Although the stimuli have identical strength, the oscillation-induced competition generates strong inhibition of the neuron receiving input from the unattended stimulus [Fig. 4(A)], which is nearly absent for the neuron whose preferred stimulus is attended [Fig. 4(B)], except at stimulus onset. As a result, the spike rates of the two neurons differ by about a factor of four, in approximate agreement with experimental results. 
(A)

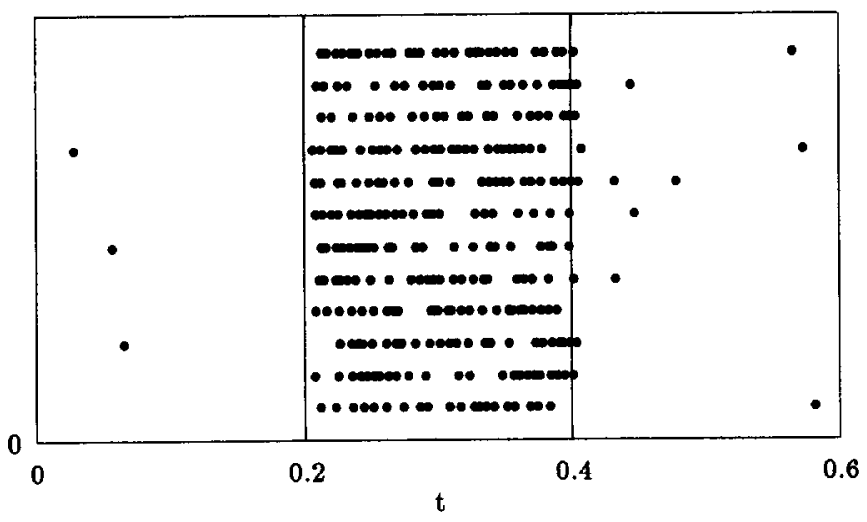

(B)

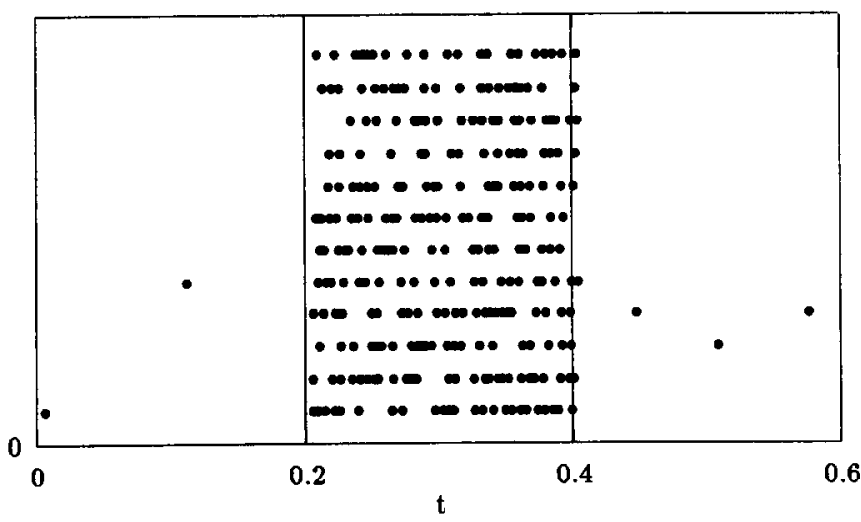

FIGURE 3. Activity in layer V1. (A) Spike trains of one cell in area V1 whose receptive field has no overlap with the focus of attention [therefore, $A=0$ in equation (2)]. Shown are 12 spike trains, each action potential being represented by a dot. In this and in (B), a stimulus is presented between $t=0.2 \mathrm{sec}$ and $t=0.4 \mathrm{sec}$. Activity outside this period is due to spontaneous firing. The frequency of the imposed oscillations is varied across runs in equal steps between 35 and $45 \mathrm{~Hz}$. The phase $\phi$ in equation (2) is chosen randomly, but remains the same during each run. (B) As for (A), but for a cell in the center of the focus of attention $\left[A=A_{\max }\right.$ in equation (2)]. The average spike rate is the same as in (A). Note the absence of any obvious periodicity.

Table 1 shows the spiking rates of pyramidal V4 neurons under different stimulus conditions.

In order to compare our simulations against the experiments of Desimone et al., we choose the analogous representation in Figs 5 and 6 to that of Fig. 10 of Desimone and Ungerleider (1989). Shown is the activity of a $\mathrm{V} 4$ neuron in response to stimulation by its preferred (here red) and non-preferred (green) stimulus. In the absence of any attentional modulation, the red bar leads to a strong neuronal response, while the green bar fails to evoke a statistically reliable response. If both stimuli are presented simultaneously in the absence of attention, the two stimuli lead to the same response as the effective stimulus alone (Table 1).

Figure 5 illustrates the influence of attention: if attention is focused on the ineffective stimulus, the mean firing rate of the cell responding to the effective stimulus is reduced by a factor of four, comparable to the reduction in firing rate reported in Moran and Desimone (1985). In other words, the receptive field shrinks around the attended stimulus. This suppression cannot be attributed to the action of an inhibitory (suppressive) penumbra around the focus of attention, since focusing attention on the ineffective stimulus fails to reduce the response to the effective stimulus as long as the attended stimulus is not inside the receptive field of the recorded cell (right hand side of Fig. 6). Therefore, attentional suppression seems to be defined in functional, not in spatial terms. This behavior is reproduced in our model.

Selecting a different part of the visual field, i.e. shifting the focus of attention from one object to another one, does not occur instantaneously. The required time has been determined in psychophysical experiments directly (Saarinen \& Julesz, 1991) as well as indirectly (Treisman $\&$ Gelade, 1980) to be in the range of $30-60 \mathrm{msec}$. These results are consistent with the electrophysiological findings that show that the time between the beginning of the shift of attention and the suppression of unattended stimuli ranges between 30 and $100 \mathrm{msec}$ (Luck, Chelazzi, Hillyard \& Desimone, 1992). We simulated a shift of attention in our model by turning off the oscillatory modulation in one part of the visual scene at a given

(A)

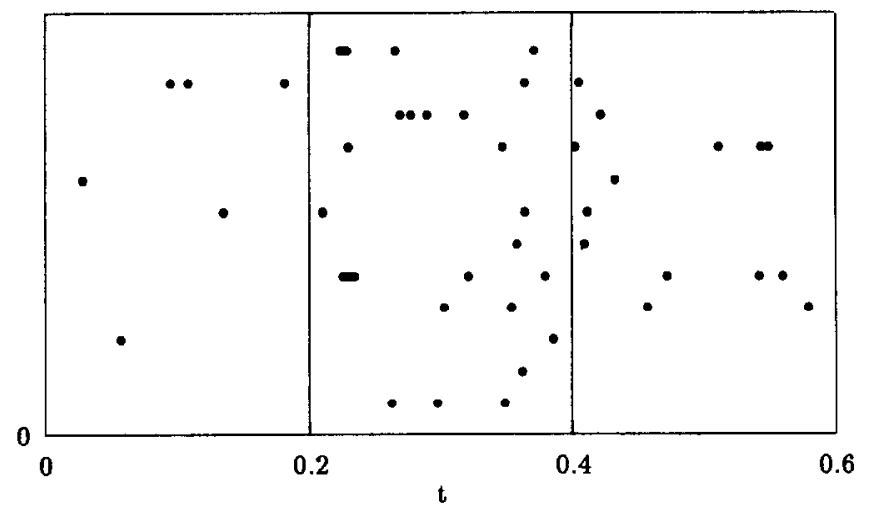

(B)

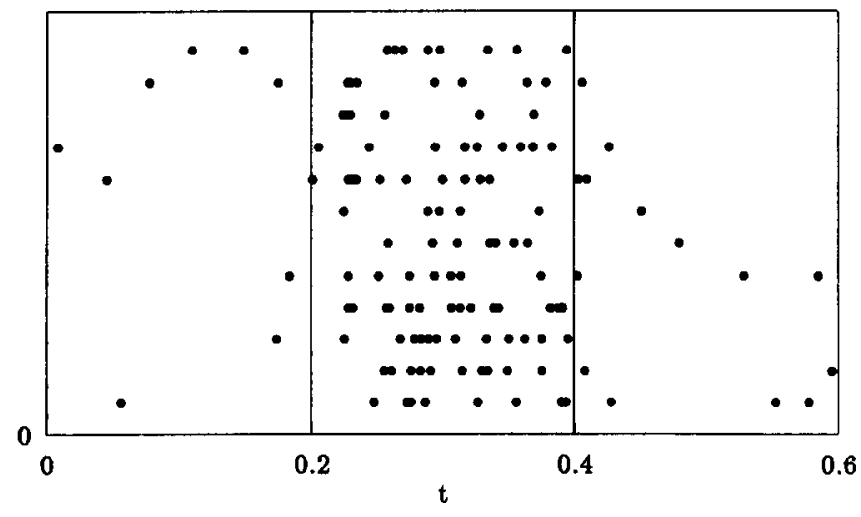

FIGURE 4. Activity in excitatory V4 cells. As in Fig. 3, the frequency of the imposed oscillations is varied across runs in equal steps between 35 and $45 \mathrm{~Hz}$ and the phase $\phi$ chosen randomly. Between $t=0.2 \mathrm{sec}$ and $t=0.4 \mathrm{sec}$, two stimuli are presented simultaneously in the receptive field of the cell, one being of the preferred kind, the other of the non-preferred kind. (A) Attention directed on the non-preferred stimulus (as in the right part of Fig. 5). (B) Attention directed on the preferred stimulus (as in the left part of Fig. 5). Note the higher firing rate in $(B)$ vs $(A)$ 

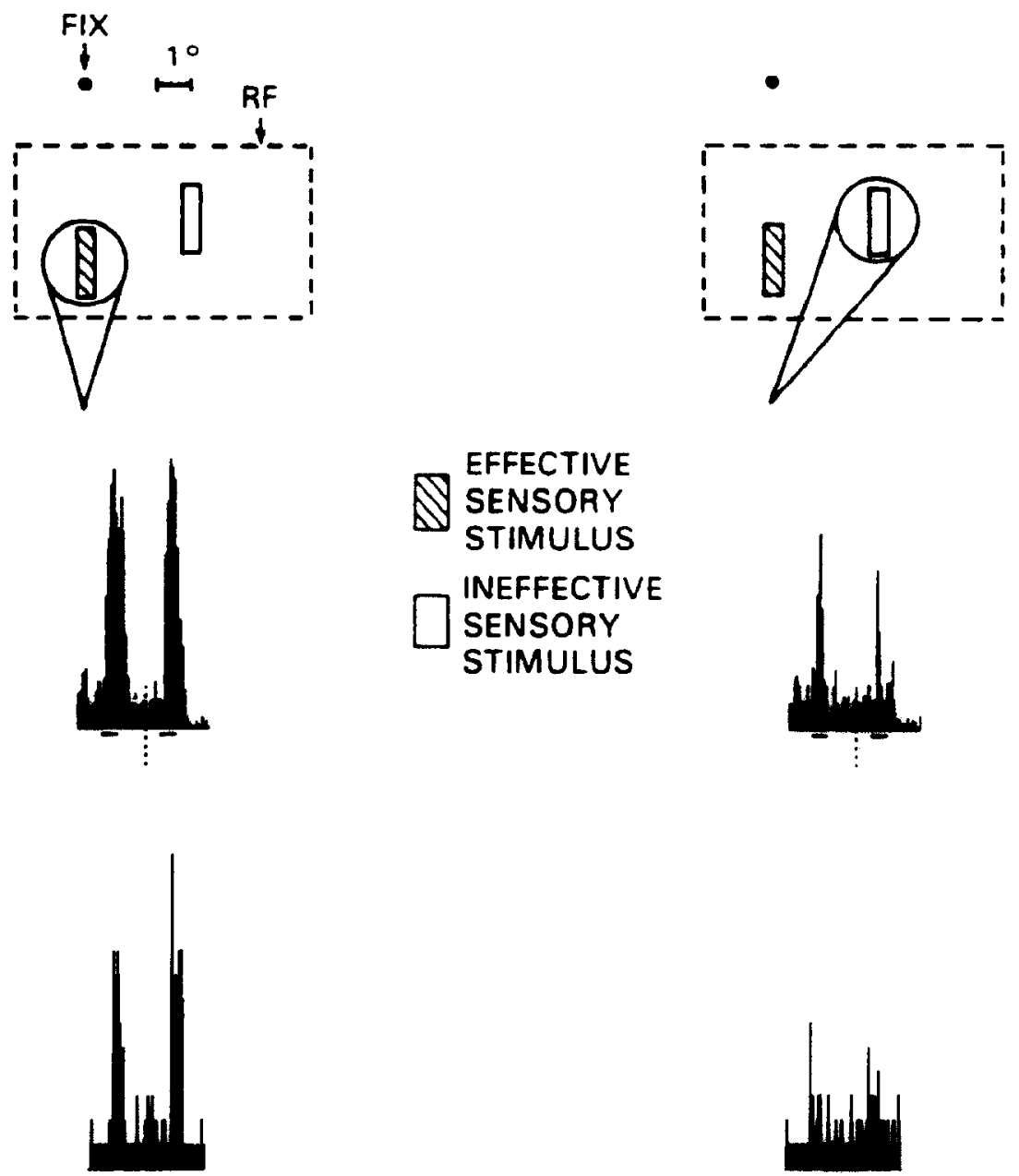

FIGURE 5. Effect of selective attention on neural response in V4 in experiment (center row) and model (bottom row). The stimulus configuration is shown in the top row. A preferred (hatched) and a non-preferred stimulus were presented simultaneously in the receptive field of the cell during two periods of length $200 \mathrm{msec}$ each, indicated by horizontal lines below the histograms. The monkey attended to one location (circled) inside the receptive fields (RF) of the cell while maintaining fixation at the fixation point (FIX). When the animal attended to the location of the effective stimulus, the cell gave a good response (left), but when the animal attended to the location of the ineffective stimulus, the cell gave only a poor response (right). Experimental data reproduced, with permission, from (Desimone \& Ungerleider, 1989); see there for details of the experimental procedure.

time, simultaneously turning it on in a different part of the scene. The underlying assumption is that the subcortical structures which generate the modulation (and which are not simulated in detail in our model) are capable of rapidly switching from one postsynaptic target population in V1 to another. We can then study the time it takes until this results in a switch in the response of neurons in area V4. Figure 7(A) shows that switching of the attentional modulation leads within $30 \mathrm{msec}$ to a change in firing rate in V4 neurons. Figure 7(B) shows that a similar delay is observed at the location which has become the new focus of attention and where the unattended stimuli have to be suppressed. Our model therefore requires a time for a shift of attention which is very similar to that found in the psychophysical and electrophysiological experiments cited above.

We analyzed our model in the frequency domain. Figure 8 shows the power spectra of two model V1 cells, one outside the focus of attention $\left[A_{\max }=0\right.$; Fig. 8(A)] and one inside $A_{\max }=0.75$; Fig. 8 . It is helpful to recall that the power spectrum of a Poisson process of mean rate $\lambda$ is given by

$$
S(f)=\lambda+2 \pi \lambda^{2} \delta(f)
$$

i.e. no particular frequency is preferred. The spectra shown in Fig. 8(A, B) exhibit two departures from this behavior. First, the power increases as a function of frequency. It has been shown (Bair et al., 1993) that this effect is due to the presence of the refractory period: Poisson distributed spike trains with a refractory period will show a dip at low frequencies. The second deviation from the Poisson behavior, present only in the case of the attended stimulus [Fig. 8(B)], is the peak around $40 \mathrm{~Hz}$, caused by the imposed frequency modulation.

This relatively subtle difference between the spectra of spike trains of attended and unattended neurons gives rise to more pronounced differences in the firing of inhibitory interneurons in extrastriate cortex. Two features distinguish the spectra of attended [Fig. 8(D)] and unattended [Fig. $8(\mathrm{C})]$ interneurons. The first is the total firing activity which is considerably smaller (by about a 
factor of five) in the unattended interneuron [Fig. 8(C)] than in the attended one [Fig. 8(D)]. The second difference is the strong peak around $40 \mathrm{~Hz}$ (accompanied by its first harmonic) in the attended neuron which has no counterpart in the unattended neuron. Note that neither of the spectra resembles that of a Poisson process very closely. This is not to be expected, however, since their internal dynamics [equations (5) and (6)] cannot be represented by a Poisson process.

The power spectra of the extrastriate pyramidal cells are shown in Fig. 8(E, F) for the non-attended and the attended case. Because the attention-driven frequency modulation of $\mathrm{V} 1$ neurons does not cause the mean rate of firing of V1 cells to increase, both V4 cells receive overall the same amount of excitatory input from V1 neurons. Yet their responses to these two cases differ substantially due to the different inhibitory inputs they receive. Their spectra resemble that of Poisson processes, with the exception of a prominent maximum between 35 and $45 \mathrm{~Hz}$ in the case of the attended neuron and a minor peak in the spectrum of the unattended neuron. The importance of the $40 \mathrm{~Hz}$ peak in the power spectrum of the attended neuron is that it allows the system to continue to use "temporal tagging" via frequency modulation to effect the same attentional gating at the next cortical site, inferotemporal cortex (IT). Thus, if two objects are located within an IT receptive fields (which can cover a substantial fraction of the entire visual scene), and the monkey is attending to one of them, interneurons in IT can pick up the frequency modulation of the V4 afferent neurons and inhibit the IT cell selective to the non-attended stimulus. Such hierarchies can extend possibly throughout the entire visual system.

The power spectra of spike trains from V4 cells show no rise in amplitude with increasing frequency (different from the spectra of V1 cells), because the firing rates of the latter are considerably smaller (around $50 \mathrm{spikes} / \mathrm{sec}$, vs around 200 in the former). If no attentional modulation is present in V4 neurons, their power spectra are completely flat (data not shown).

\section{DISCUSSION}

We here simulate the Crick and Koch (1990b) model for the neuronal implementation of selective visual attention based on the temporal modulation of sensory
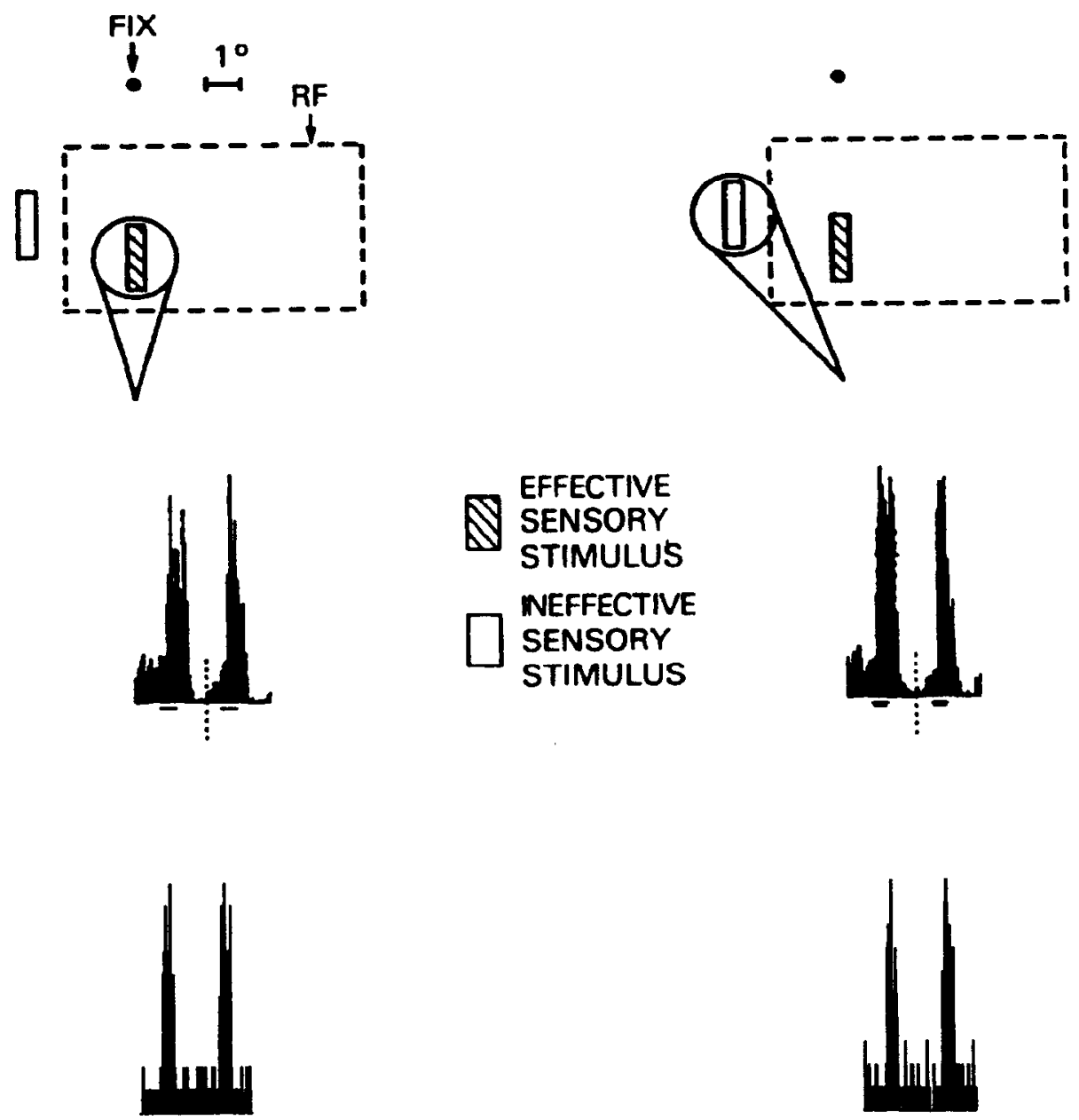

FIGURE 6. Response of a V4 cell with only one stimulus present in its receptive field, experimental data (center row) and simulation results (bottom row); symbols as in Fig. 5 . In the experiment shown to the left, the monkey attends to the object inside the receptive field, while in the case shown to the right, the attention is directed to an object outside the receptive field. In both cases, the cell response is comparable to the response to two stimuli when the monkey attended the preferred stimulus (Fig. 5, left) and significantly larger than to the case when the monkey attended the non-preferred of two stimuli (Fig. 5, right). 
evoked activity in striate cortex. This modulation is used to label neurons responding to properties of an "attended" object and enables the system to suppress the activity to non-attended objects present in close neighborhood of the "attended" object. The model is compatible with the known anatomy of the mammalian visual system and makes use of simple single neuron models: neurons in V1 generate spikes with a Poisson distribution (with either a constant or a time-varying mean rate of fire) while pyramidal cells in V4 are leaky-integrate and fire cells. The only non-standard elements are the inhibitory V4 neurons with bandpass characteristics.

Because no evidence exists that attention acts to boost the firing rates of neurons in primary visual cortex responding to the attended object (or reduces the firing frequency of neurons responding to other stimuli), Crick and Koch (1990b) proposed that attention labels the temporal structure of the neuronal discharge, for instance by imposing an oscillatory pattern onto the discharge, similar to that observed by a number of researchers in cat and monkey visual cortex (Gray \& Singer, 1989; Eckhorn et al., 1988; Kreiter \& Singer, 1992; Livingstone, 1991). This temporal signal must then be "demodulated" by neurons in V4. For this purpose we introduce neurons with bandpass properties, similar to the haircells in the amphibian cochlea which optimally respond only to a band of input frequencies (Crawford \& Fettiplace, 1981; Fettiplace, 1987). Rather than modeling the detailed conductances underlying this behavior (Hudspeth \& Lewis, 1988b), we choose a more generic approach, using a circuit description of the neuronal membrane of these cells in terms of RLC elements derived by linearization around the resting potential (Koch, 1984). Such neurons are expected to generate damped oscillations in the $40 \mathrm{~Hz}$ range when excited and, indeed, several classes of (presumed inhibitory) interneurons with oscillatory behavior in this frequency range have been described in layer 4 of guinea pig sensory cortex (Llinas et al., 1991). In our model, these cells project to pyramidal cells, and, indeed, $40 \mathrm{~Hz}$ inhibitory postsynaptic potentials have been identified during in vivo intracellular recordings in cat area 17 pyramidal cells (Ferster, 1988).

Our model ties together the experimental observation of the influence of selective attention on the firing rates in areas V4 and higher, but not in V1 (Moran \& Desimone, 1985), with that of $40 \mathrm{~Hz}$ oscillations in primary visual cortex. One noteworthy property of these oscillations is that they are stimulus-induced (i.e. they only appear when a stimulus is present) but not stimuluslocked (i.e. they do not have a strict phase relation with the stimulus onset) and therefore disappear after averaging over stimuli. The oscillations in our model have the same properties.

Despite the simplicity of the model, it is capable of reproducing electrophysiological and psychophysical results quantitatively (Figs 5, 6, and 7, respectively). Noise in the form of spontaneous activity was included in all stages to demonstrate the robustness of the proposed mechanism. The data in Figs 5 and 6 were obtained with
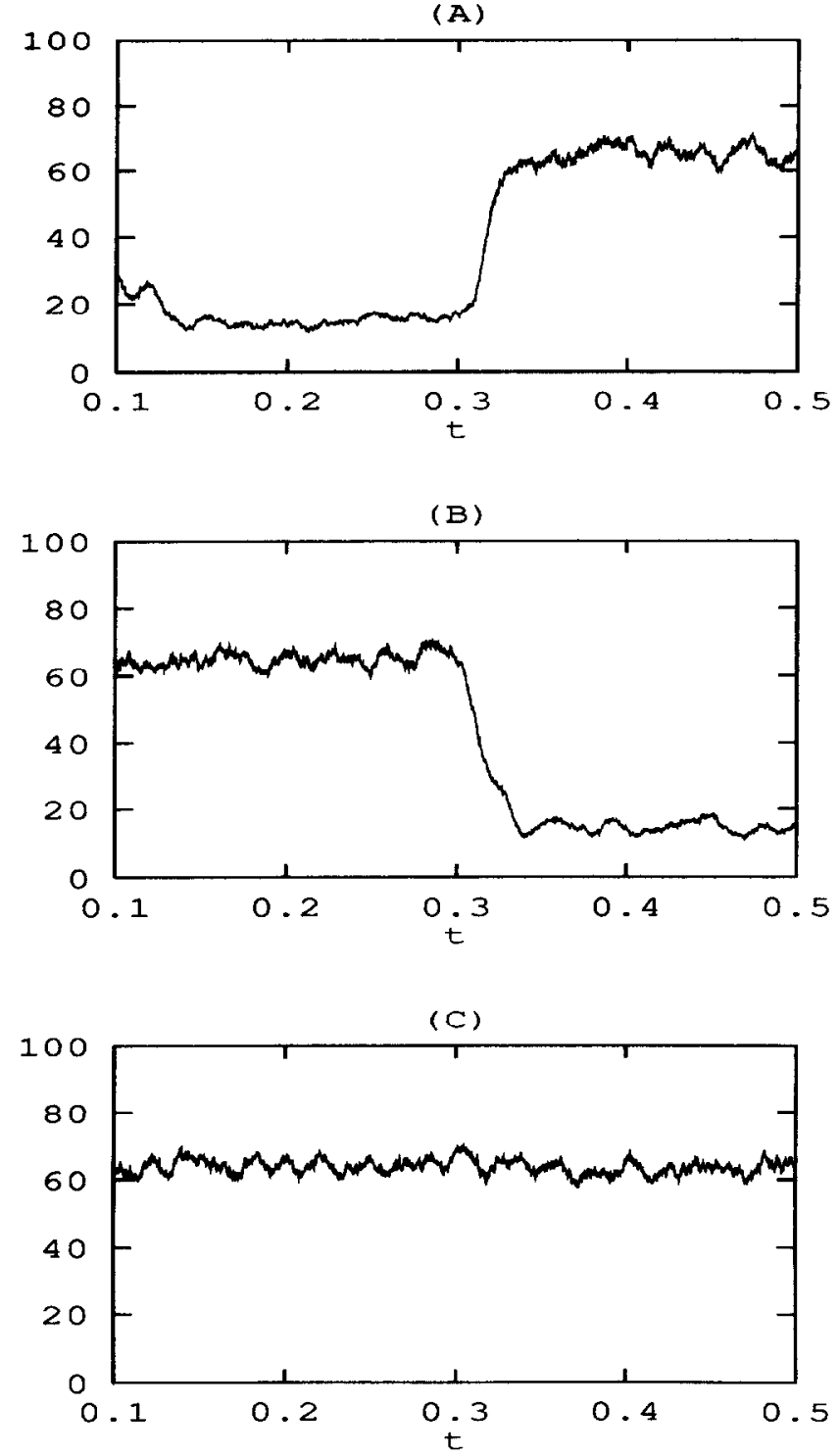

FIGURE 7. Shifting the focus of attention. Shown are the averaged firing rates of three different pyramidal cells in V4. (A) Cell whose receptive field contains both a preferred and a non-preferred stimulus, with attention focused on the non-preferred stimulus (right hand side of Fig. 5). At $t=0.3 \mathrm{sec}$, attention is shifted to a stimulus outside the cell's receptive field. The firing rate stabilizes within $30 \mathrm{msec}$ to its new value. (B) Here, attention is shifted from a location outside the receptive field to the non-preferred stimulus inside the receptive field. The shift of attention occurs at $t=0.3 \mathrm{sec}$ and the new value is attained within $30 \mathrm{msec}$, mimicking the times estimated using a psychophysical paradigm for shifting the focus of attention (Saarinen \& Julesz, 1991). (C) As in (B), but now the attention is shifted to the preferred stimulus inside the receptive field. It is seen that no significant change in the firing rate occurs when the attention is shifted (at $t=0.3 \mathrm{sec}$ ) or thereafter, i.e. the attentional state does not alter the firing rate induced by an efficient stimulus. All data are averaged over 1000 runs with randomly chosen $\phi$ in equation (2) and a frequency uniformly distributed between 35 and $45 \mathrm{~Hz}$.

the frequency imposed on the $\mathrm{VI}$ neurons varied in the range $40 \pm 5 \mathrm{~Hz}$, to show that no very sharp frequency tuning is required for the functioning of the model.

Our model makes specific predictions. In particular, both V1 and V4 pyramidal cells should have $40 \mathrm{~Hz}$ local maxima in their power spectra as long as the attended object is within their receptive field [Fig. 8(A, F)]. Furthermore, given the bandpass nature of the inhibitory 
(A)

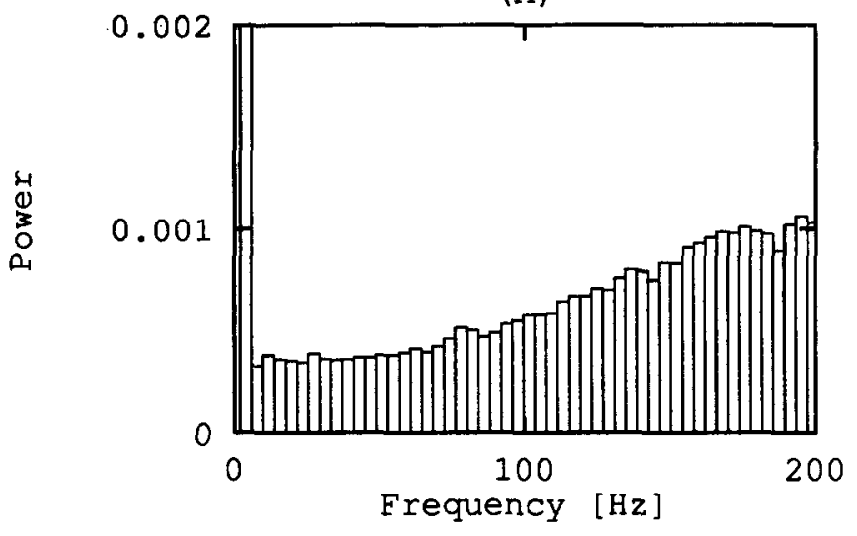

(C)

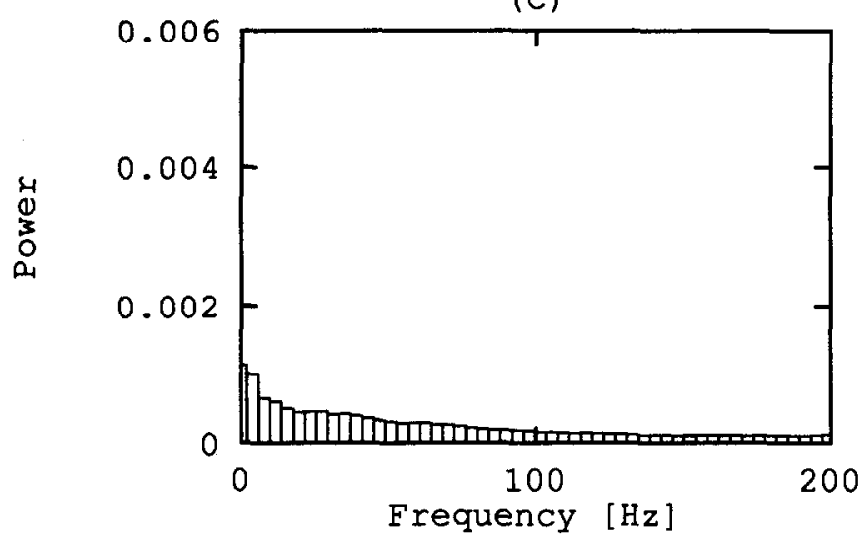

(E)

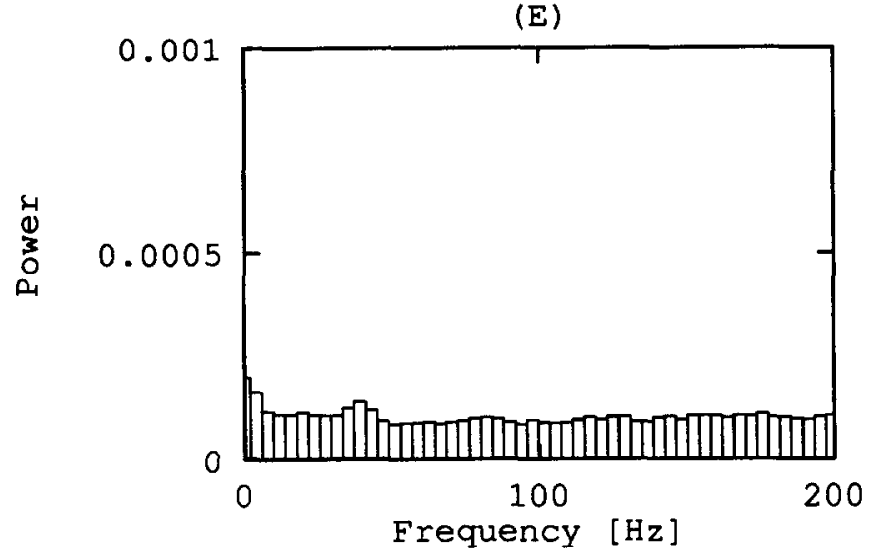

(B)

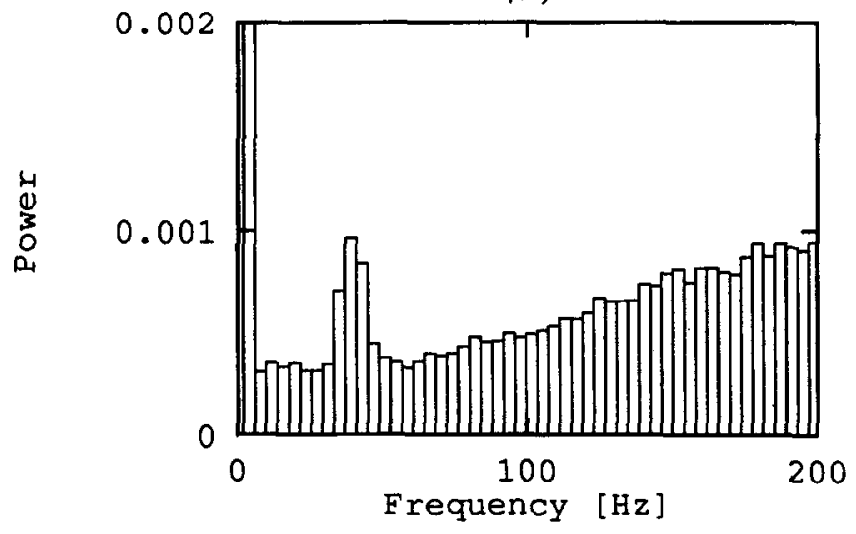

(D)

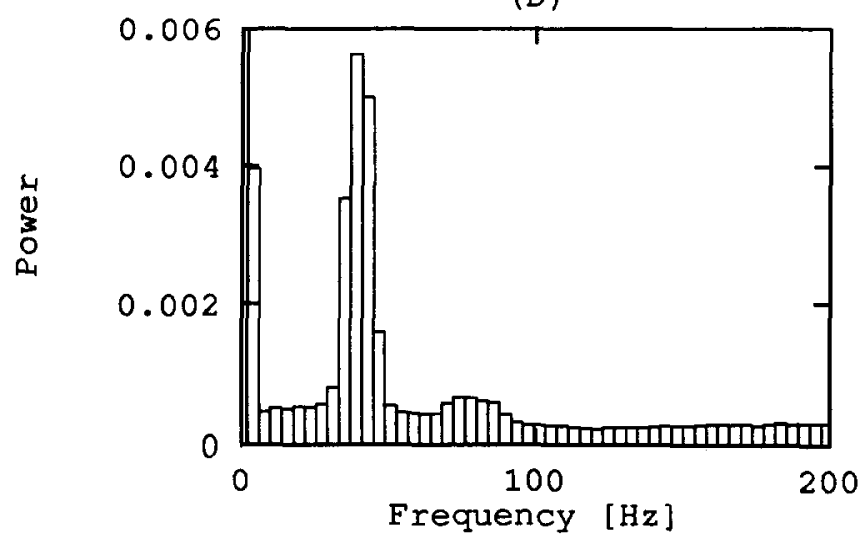

(F)

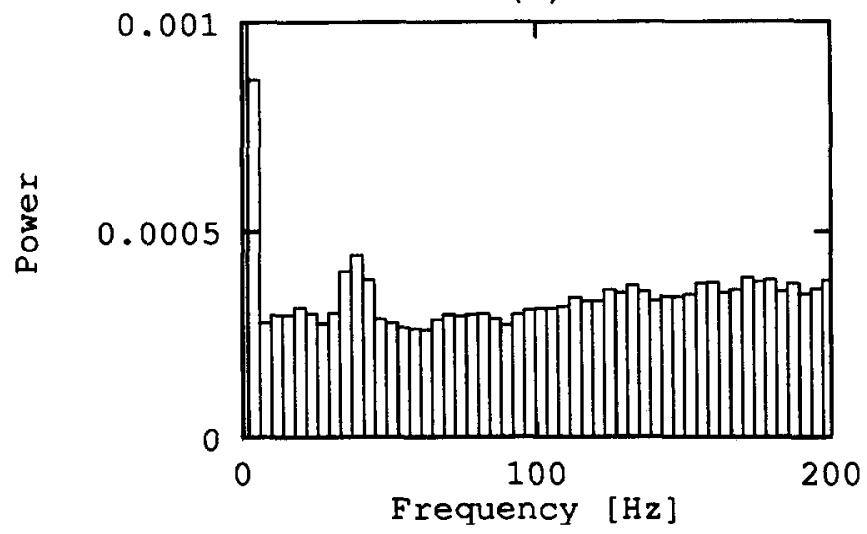

FIGURE 8. The power spectra of the firing frequency response of V1 and V4 cells. The Fourier transform of the power spectrum is identical to the autocorrelation function commonly used by electrophysiologists (e.g. Gray \& Singer, 1989). All data are averaged over 64 runs of $1024 \mathrm{msec}$ each; the spectra are computed using the FFT routine; the frequency imposed on attended V1 neurons was varied across trials in equal steps between 35 and $45 \mathrm{~Hz}$. (A) The spectrum of a V1 cell while attention is outside its receptive field $\left(A_{\max }=0\right)$ and $(\mathrm{B})$ the spectrum while the spotlight of attention overlaps with its receptive field $\left(A_{\max }=0.75\right)$. In comparison, the spectrum of a pure Poisson process of rate $\lambda$ is constant (of amplitude $\lambda$ ) with a delta peak at the origin, corresponding to our intuitive notion that a Poisson process favors no particular frequency. A Poisson process with refractory period shows a dip at low frequencies (Bair et al., 1993). The action of attention is to impose a peak between 35 and $45 \mathrm{~Hz}$. The spectra of the inhibitory V4 cells are shown in (C) without attention and in (D) with oscillatory input. Our model predicts that these cells show a very large peak in the $40 \mathrm{~Hz}$ range [a second harmonic around $80 \mathrm{~Hz}$ is evident in (D)]. The power spectra of the excitatory V4 cells are shown in (E) without and in (F) with attention. Notice the three-fold increase in the mean level of the spectrum when going from the non-attended to the attended situation [i.e. from (E) to (F)]. The $40 \mathrm{~Hz}$ peak is approximately as strong here as in an attended V1 cell, allowing the same temporal "tagging" mechanism to be repeated between V4 and its next cortical target IT, in agreement with the experiments of Moran and Desimone (1985). 
cells, their firing pattern should show a large peak at $40 \mathrm{~Hz}$ during the same condition [Fig. 8(D)]. Llinas et al. (1991) reported that the cortical interneurons with oscillatory activity they observed are small and hard to find and would be discriminated against large pyramidal cells [which have less oscillatory activity; Fig. 8(E, F)] in micro-electrode recordings. If the system is attending a location devoid of any stimulus, V1 and V4 cells would fire at their spontaneous level and no oscillatory activity will be noted.

We show that moderate levels of modulation (i.e. $A_{\max }=0.75$ ) are sufficient in our very crude model to mimick the experimental result. If a mechanism similar to the one proposed here is, in fact, used in the attentional system, it may be expected that specialized cells can make use of much smaller levels of frequency modulation levels. Therefore, very careful studies of the detailed temporal structure of neural signals will be necessary to detect the presence of such a modulatory discharge in single trials. Furthermore, although the experiments in cats (Gray \& Singer, 1989) and monkeys (Kreiter \& Singer, 1992; Livingstone, 1991; Murthy \& Fetz, 1992) clearly show the presence of semisynchronous cortical oscillations in the $25-65 \mathrm{~Hz}$ range, decisive evidence for or against our model would have to come from experiments in which the attentional state of the animal is carefully controlled and in which cell behavior inside and outside the focus of attention of trained monkeys is monitored. Oscillations are only expected in our model for neurons whose receptive fields overlaps with the focus of attention, usually a small fraction of the total visual field. It is well possible that the failure of two recent studies to detect $40 \mathrm{~Hz}$ oscillations in single and multi-unit as well as local field potentials in the anesthetized and the awake and behaving monkey in areas V1, MT and IT (Tovee \& Rolls, 1992; Young et al., 1992) may simply reflect the absence of attentional modulation during these experiments.

Desimone (1992) suggests a classification of neuronal models of attention in two categories, providing examples for both types of models. In his terminology, our model belongs to the "cell-gating" class, because the activity of cells is influenced directly. He outlined a cell-gating model which is also based on competition between V4 cells with preference for different features. As in our model, he suggests that attention biases the competition in favor of the attended stimuli, but his model is less detailed and does not specify the "biasing" or "labeling" mechanism.

Models from Desimone's second category are called "input-gated", because the cells' input along the occipitotemporal pathway is modified. An example of this class is the shifter model of attention proposed by Olshausen et al. (1993). In this model, the key action of attention is to selectively map (or "route") parts of the visual scene onto cells in V4 and IT. This mapping (using specialized "shifter" circuits) preserves spatial relationships within the window of attention. Only signals within the attentional spotlight are transmitted onto higher cortical areas.
In our language, attentional "labeling" is carried out by modulating the mean discharge rate (down to zero in the case of signals outside the window of attention). Their model is compatible with the Moran and Desimone (1985) data (see Fig. 18 in Olshausen et al., 1993) but quite distinct from our model which assumes no such preservation of spatial relationships.

We here use synchronous oscillations as a tagging mechanism, but would like to point out that other temporal modulation patterns may work as well. For instance, the action of the saliency map could be to synchronize a group of neurons in striate cortex when their receptive fields overlap with the focus of attention (Niebur \& Koch, 1993). While the mean rate of firing of V1 cells would not vary with attention, all neurons within the "spotlight" would have a higher probability of firing simultaneously. It is clear that this typc of temporal synchronization (Milner, 1974; von der Malsburg \& Schneider, 1986) could not be detected in any single-neuron spike train but only in multi-unit recordings of neurons inside and outside the focus of attention.

Due to the distributed coding of features in cortex, a particular stimulus will induce neural responses at different cortical locations. For instance, in oversimplified terms, the motion of the object will activate neurons in area MT, its hue neurons in V4 etc. This is not limited to features within one modality: if an object emits an auditory signal, it will generate activity in area $\mathrm{A} 1 \mathrm{etc}$.

This distributed coding scheme has consequences not only for the specific problem of the control of selective attention but also, more generally, for the basic mechanisms of perception. Concerning selective attention, it has to be ensured that at all levels in the cortical hierarchy, the stimuli selected for close scrutiny can be distinguished from other stimuli impinging on the sensory system. A simple mechanism for accomplishing this would be direct suppression of the non-selected stimuli or enhancement of the selected stimuli, carried out by direct neural connections from a central structure (or multiple structures) to the areas to be modulated. Given the multitude of anatomical connections between cortex and associated subcortical nuclei, and between different areas of the cortex, such a model cannot be ruled out conclusively at our present rudimentary state of insight in the function of these connections. Nevertheless, this solution would require very substantial wiring across nearly all parts of the brain which are involved in perception and cognition. One decisive advantage of a mechanism such as ours is its parsimony: tagging requires only anatomical connections from the control structures to the primary sensory structures (not to all the higher areas), and the attentional modulation is then multiplexed on the neural signals. The tag is carried by the neural signals through the whole hierarchy and is available at all higher stages. Therefore, the same connections are used for conveying information about the stimulus qualities and about the attentional modulation of the input. We believe that this is a much more efficient use of the limited connectivity resources than direct modulation of all involved areas. 
The second consequence of the distributed coding of features is that the brain must find a way to identify and distinguish objects based on the conjunction of features. It is clear that there cannot exist a specialized neuron for every person or object that may appear at some time or other in the visual field. Psychophysical evidence (Treisman \& Gelade, 1980) indicates that focal attention is necessary to solve this "binding problem". In the Crick and Koch model $(1990 \mathrm{a}, \mathrm{b})$, this is achieved naturally by imprinting a temporal structure on the neural signals at the peripheral level, which can then be detected in all structures higher in the cortical hierarchy. The representations of features of the objects inside the focus of attention share a common trait throughout all cortical areas, namely their temporal structure, while this is not the case for objects outside the focus of attention.

\section{REFERENCES}

Bair, W., Koch, C., Newsome, W. \& Britten, K. (1993). Power spectrum analysis of MT neurons in the awake monkey. In Bower, J. \& Eeckman, F. (Eds), Computation and neural systems. Norwell, Mass.: Kluwer.

Bernander, O., Douglas, R., Martin, K. \& Koch, C. (1991). Synaptic background activity influences spatiotemporal integration in single pyramidal cells. Proceedings of the National Academy of Science, U.S.A., 88, 11569-11573.

Cauller, L. J. \& Connors, B. W. (1992). Functions of very distal dendrites: Experimental and computational studies of layer $I$ synapses on neocortical pyramidal cells. In McKenna, T., Davis, J. \& Zornetzer, S. F. (Eds), Single neuron computation (pp. 199-229). San Diego, Calif: Academic Press.

Colby, C. L. (1991). The neuroanatomy and neurophysiology of attention. Journal of Child Neurology, 6, \$90-\$118.

Crawford, A. C. \& Fettiplace, R. (1981). An electrical tuning mechanism in turtle cochelar hair cells. Journal of Physiology, London, 312, $377-412$.

Crick, F. \& Koch, C. (1990a). Some reflections on visual awareness. Cold Spring Harbor Symposiums in Quantitative Biology, $L V, 953-962$.

Crick, F. \& Koch, C. (1990b). Towards a neurobiological theory of consciousness. Seminars in the Neurosciences, 2, 263-275.

Desimone, R. (1992). Neural circuits for visual attention in the primate brain. In Carpenter, G. \& Grossber, S. (Eds), Neural networks for vision and image processing. Cambridge, Mass.: MIT Press.

Desimone, R. \& Ungerleider, L. G. (1989). Neural mechanisms of visual processing in monkeys. In Boller, F. \& Graffman, J. (Eds), Handbook of neuropsychology (pp. 267-299). Amsterdam: Elsevier.

Desimone, R., Wessinger, M., Thomas, L. \& Schneider, W. (1989). Effects of deactivation of lateral pulvinar or superior colliculus to selectively attend to a visual stimulus. Society of Neurascience Abstracts, 15,162

Desimone, R., Wessinger, M., Thomas, L. \& Schneider, W. (1991). Attentional control of visual perception: Cortical and subcortical mechanisms. Cold Spring Harbor Symposiums in Quantitative Biology, $L V, 963-971$.

Eckhorn, R., Bauer, R., Jordan, W., Brosch, M., Kruse, W., Munk, M. \& Reitboeck, H. J. (1988). Coherent oscillations: A mechanism of feature linking in the visual cortex? Biological Cybernetics, 60 , 121-130.

Ferster, D. (1988). Spatially opponent excitation and inhibition in simple cells of the cat visual cortex. Journal of Neuroscience, 8 , 1172-1180.

Fettiplace, R. (1987). Electrical tuning of hair cells in the inner ear. Trends in Neurasciences, 10, 421-425.

Garey, L. J., Dreher, B. \& Robinson, S. R. (1991). The organization of the visual thalamus. In Dreher, B. \& Robinson, S. R. (Eds), Neuroanatomy of the visual pathways and their development (pp. 176-234). Boca Raton, Fla.: CRC Press.
Gray, C. M. \& Singer, W. (1989). Stimulus-specific neuronal oscillations in orientation columns of cat visual cortex. Proceedings of the National Academy of Sciences, U.S.A., 86, 1698-1702.

Hudspeth, A. J. \& Lewis, R. S. (1988a). Kinetic analysis of voltageand ion-dependent conductances in saccular hair cells of the bull frog, Rana catesbeiana. Journal of Physiology, London, 400 , 237-274.

Hudspeth, A. J. \& Lewis, R. S. (1988b). A model for electrical resonance and frequency tuning in saccular hair cells of the bull-frog, Rana catesbeiana. Journal of Physiology, London, 400, 275-297.

Hutcheon, B. \& Puil, E. (1992). A low-frequency subthreshold resonance in neocortical neurons generated mainly by $\mathrm{Ih}$. Society of Neuroscience Abstracts, $18,1344$.

Jones, E. G. (1985). The thalamus. New York: Plenum Press.

Julesz, B. (1991). Early vision and focal attention. Reviews of Modern Physics, 63, 735-772.

Kanwisher, N. \& Driver, J. (1992). Objects, attributes and visual attention: Which, what, and where. Current Directions in Psychological Science, 1, 26-31.

Koch, C. (1984). Cable theory in neurons with active, linearized membranes. Biological Cybernetics, 50, 15-33.

Koch, C. \& Ullman, S. (1985). Shifts in selective visual attention: Towards the underlying neural circuitry. Human Neurobiology, 4, 219-227.

Kreiter, A. K. \& Singer, W. (1992). Oscillatory neuronal responses in the visual-cortex of the awake macaque monkey. European Journal of Neuroscience, 4, 369-375.

Kubota, T., Morimoto, M., Kanaseki, T. \& Inomata, H. (1988). Visual pretectal neurons projecting to the dorsal lateral geniculate nucleus and pulvinar nucleus in the cat. Brain Research Bulletin, 20 , 573-579.

LaBerge, D. \& Buchsbaum, M. S. (1990). Positron emission tomographic measurements of pulvinar activity during an attention task. Journal of Neuroscience, 10, 613-619.

Livingstone, M. S. (1991). Visually-evoked oscillations in monkey striate cortex. Society of Neuroscience Abstracts, 17, 176

Llinas, R. R., Grace, A. A. \& Yarom, Y. (1991). In vitro neurons in mammalian cortical layer 4 exhibit intrinsic oscillatory activity in the 10- to $50-\mathrm{Hz}$ frequency range. Proceedings of the National Academy of Sciences, U.S.A., 88, 987-901.

Luck, S. J., Chelazzi, L., Hillyard, S. A. \& Desimone, R. (1992). Attentional modulation of responses in area $\mathrm{V} 4$ of the macaque. Society of Neuroscience Abstracts, 18, 147.

von der Malsburg, C. \& Schneider, W. (1986). A neural cocktail party processor. Biological Cybernetics, 54, $29-40$.

Mauro, A., Conti, F., Dodge, F. \& Schor, R. (1970). Subthreshold behavior and phenomenological impedance of the squid giant axon. Journal of General Physiology, 55, 497-523.

Milner. P. A. (1974). A model for visual shape recognition. Psychological Review, 81, 521-535.

Moran, J. \& Desimone, R. (1985). Selective attention gates visual processing in the extrastriate cortex. Science, 229, 782-784.

Murthy, V. N. \& Fetz, E. E. (1992). Coherent 23- to $35 \mathrm{~Hz}$ oscillations in the sensorimotor cortex of awake behaving monkey. Proceedings of the National Academy of Science, U.S.A., 89, 5670-5674.

Niebur, E. \& Koch, C. (1993). A model of the physiological basis of selective attention based on the temporal structure of neural signals. Society of Neuroscience Abstracts, 19.

Olshausen, B., Andersen, C. \& Van Essen, D. (1993). A neural model of visual attention and invariant pattern recognition. Journal of Neuroscience. In press.

Petersen, S. E., Robinson, D. L. \& Morris, J. D. (1987). Contributions of the pulvinar to visual spatial attention. Neuropsychologia, 25, 97-105.

Posner, M. I. \& Driver, J. (1992). The neurobiology of selective attention. Current Opinion in Neurobiology, 2, 165-169.

Posner, M. I. \& Petersen, S. E. (1990). The attention system of the human brain. Annual Review of Neuroscience, 13, 25-42.

Rubinson, D. L. \& Petersen, S. E. (1992). The pulvinar and visual salience. Trends in Neurosciences, 15, 127-132.

Saarinen, J. \& Julesz, B. (1991). The speed of attentional shifts in the visual field. Proceedings of the National Academy of Sciences, U.S.A. $88,1812-1814$ 
Softky, W, \& Koch, C. (1993). The highly irregular firing of cortical-cells is inconsistent with temporal integration of random epsps. Journal of Neuroscience, 13, 334-350.

Tovee, M. J. \& Rolls, E. T. (1992), Oscillatory activity is not evident in the primate temporal visual cortex with static stimuli. NeuroReport, 3 , 369-372.

Treisman, A. (1988). Features and objects: The fourteenth Bartlett memorial lecture. Quantitative Journal of Experimental Psychology, 40A, 201-237.

Treisman, A. \& Gelade, G. (1980). A feature-integration theory of attention. Cognitive Psychology, 12, 97-136.

Yamada, W., Koch, C. \& Adams, P. R. (1989), Multiple channels and calcium dynamics. In Koch, C. \& Segev, I. (Eds), Methods in neuronal modeling: From synapses to networks (pp. 97-134). Cambridge, Mass.: MIT Press.
Young, M. P., Tanaka, K. \& Yamane, S. (1992). On oscillating neuronal responses in the visual cortex of the monkey. Journal of Neurophysiol ogy, 67, 1464-1474.

Acknowledgements - This work was supported by the Office of Naval Research, the Air Force Office of Scientific Research, the National Science Foundation, and the James S. McDonnell Foundation. The authors wish to acknowledge the Advanced Computing Laboratory of Los Alamos National Laboratory, Los Alamos, NM 87545. Some of the numerical work was performed on computing resources located at this facility. We thank Francis Crick for helpful discussions, Bob Desimone for providing us with parts of Figs 5 and 6, Wyeth Bair for writing the routines for the spectral analysis, and Marius Usher for a critical reading of the manuscript. 